\title{
OPERANT BEHAVIOR TO OBTAIN PALATABLE FOOD MODIFIES NEURONAL PLASTICITY IN THE BRAIN REWARD CIRCUIT
}

Thomas Guegan ${ }^{1}$, Laura Cutando ${ }^{1}$, Eduard Ayuso ${ }^{4}$, Emanuela Santini ${ }^{2}$, Gilberto Fisone

${ }^{2}$, Fatima Bosch ${ }^{4}$, Albert Martinez ${ }^{3}$, Emmanuel Valjent ${ }^{2 \#}$, Rafael Maldonado* ${ }^{1} \&$ Miquel Martin* ${ }^{1}$

1 Laboratori de Neurofarmacologia, Univeristat Pompeu Fabra, PRBB, Barcelona, Spain.

${ }^{2}$ Department of Neuroscience, Karolinska Institutet, Stockholm, Sweden.

${ }^{3}$ Departament de Biologia Cel.lular, Universitat de Barcelona/ Institut de Recerca en Biomedicina, Barcelona/CIBERNED, Spain.

${ }^{4}$ Center of Animal Biotechnology and Gene Therapy and Department of Biochemistry and Molecular Biology, School of Veterinary Medicine, Universitat Autònoma de Barcelona, 08193-Bellaterra, Spain.

\# Current address: IGF. Department of Neurobiology Inserm U661, UMR 5203 CNRS, University Montpellier I \& II ; 141, rue de la Cardonille, 34094 Montpeller Cedex 05France

* Corresponding authors:

Rafael Maldonado and Miquel Martín.

Laboratori de Neurofarmacologia.

Universitat Pompeu Fabra, Parc de Recerca Biomedica de Barcelona

C/ Dr. Aiguader 88, 08003, Barcelona, Spain.

Telf: +34933160824ＦA: +34933160901

E-mail address: $\underline{\text { rafael.maldonado@upf.edu and miquel.martin@upf.edu }}$ 


\begin{abstract}
Palatability enhances food intake by hedonic mechanisms that prevail over caloric necessities. Different studies have demonstrated the role of the endogenous cannabinoids in the mesocorticolimbic system in controlling food hedonic value and consumption. We hypothesize that the endogenous cannabinoid system could also be involved in the development of food-induced behavioral alterations, such as foodseeking and binge-eating, by a mechanism that requires neuroplastic changes in the brain reward pathway. For this purpose, we evaluated the role of the CB1 cannabinoid receptor $(\mathrm{CB} 1-\mathrm{R})$ in the behavioral and neuroplastic changes induced by operant training for standard, high caloric or high palatable food using different genetics, viral and pharmacological approaches. Neuroplasticity was evaluated by measuring changes in dendritic spine density in neurons previously labeled with the dye DiI. Only operant training to obtain high-palatable food induced neuroplastic changes in neurons of the nucleus accumbens shell and prefrontal cortex that were associated to changes in foodseeking behavior. These behavioral and neuroplastic modifications induced by palatable food were dependent on the activity of the CB1-R. Neuroplastic changes induced by palatable food are similar to those produced by some drugs of abuse and may be crucial in the alteration of food-seeking behavior leading to overweight and obesity.
\end{abstract}

Keywords: CB1 cannabinoid receptor, food-seeking, mesocorticolimbic, palatability, structural plasticity. 


\section{Introduction}

The large availability to high palatable food is a crucial factor that promotes overeating in developed countries (Saper et al., 2002) and finally can lead to overweight, obesity and associated illnesses. The prevalence of obesity has increased substantially since the mid-twentieth century in virtually every country (Caballero, 2007), and obesity prevalence in adults has doubled in the US since 1980, where $34 \%$ of US adults are obese (Khan et al., 2009).

Overeating behavior shares similarities with the loss of control and compulsive taking behavior observed in drug-addicts. Thus, deficiencies in inhibitory capacity have been reported in obese humans with excessive food intake (Bederkoorn et al., 2006; Rosval et al., 2006) that are similar to the elevated impulsivity leading to relapse in drug addicts (Moeller et al., 2006). In addition, overconsumption of high caloric food produces neuroadaptive changes in the brain reward system that can drive the development of compulsive eating (Johnson and Kenny, 2010). Food, like drugs of abuse, activates the mesocorticolimbic system, which mediates the hedonic and motivational aspects of different rewarding stimuli. Repeated activation of this brain pathway may lead to neuroadaptive changes and structural reorganization that could participate in the behavioral alterations promoted by drugs of abuse (Russo et al., 2010). Early studies showed a correlation between changes in neuroplasticity and locomotor sensitization induced by cocaine ( $\mathrm{Li}$ et al., 2004), although more recent studies have reported conflicting results (for review see Russo et al., 2010). It is currently unclear whether similar neuroplastic alterations occur with repeated exposure to natural rewards. Indeed, most studies have failed to observe changes in structural plasticity in the mesocorticolimbic system induced by food (Robinson et al., 2001; Crombag et al., 
2005), although another natural reward, sex, has been recently reported to modify dendritic morphology in the reward circuit (Pitchers et al., 2010).

The endogenous cannabinoid system is a key modulator of the hedonic value of natural rewards (Di Marzo and Matias, 2005) and drugs of abuse (Maldonado et al., 2006). These rewarding responses seem to be mediated, at least in part, by the activation of CB1-R in the mesocorticolimbic system (Maldonado et al., 2006). CB1-R are also involved in mediating neuroplastic changes induced by drugs of abuse (BallesterosYañez et al., 2007). Considering the key role of CB1-R in food rewarding effects, these receptors could also be involved in any possible neuroplasticity change induced by the exposure to a natural reward.

In this study, we have investigated the ability of palatable food-induced seeking behavior to promote neuroplasticity and the possible involvement of $\mathrm{CB} 1-\mathrm{R}$ in these changes. For this purpose, CB1 knockout mice (CB1-/-) and wild-type littermates $(\mathrm{CB} 1+/+)$ were trained to lever-press to obtain standard, high caloric or high palatable pellets and the changes in morphological plasticity were evaluated. We found that operant behavior to obtain high palatable food produced changes in structural plasticity in specific areas of the mesocorticolimbic system. These changes were absent in CB1-/and in $\mathrm{CB} 1+/+$ with a reduced $\mathrm{CB} 1-\mathrm{R}$ activity in the nucleus accumbens (NAc). 


\section{Experimental procedures}

\subsection{Animals}

The experiments were carried out in male $\mathrm{CB} 1-/-$ and $\mathrm{CB} 1+/+$ littermates from $8-$ 12 weeks old at the beginning of the experiments. The generation of CB1-/- and CB1+/+ was described previously (Zimmer et al., 1999). Briefly, homozygous CB1+/+ and CB1-/- were bred by back-crossing of chimeric and heterozygous animals to C57BL6/J and interbreeding of heterozygous animals for at least 10 generations in order to obtain a pure C57BL6/J background. The animals were individually housed and maintained in a controlled temperature $\left(21 \pm 1^{\circ} \mathrm{C}\right)$ and humidity $(55 \pm 10 \%)$ room with a 12:12-h reversed light/dark cycle (on at 8 p.m and off at 8 a.m.). All the experiments were performed during the dark phase of the dark/light cycle. Animals were habituated to the experimental room and handled for one week before starting the experiments with ad libitum access to standard chow and water. All animal procedures were conducted in accordance with the standard ethical guidelines (European Communities Directive 86/60-EEC, Animal Welfare Assurance \#A5388-01, IACUC Approval Date 06/08/2009) and approved by the local ethical committee (Comitè Ètic d'Experimentació Animal-Institut Municipal d'Assistència Sanitària-Universitat Pompeu Fabra).

\subsection{Acquisition of operant responding maintained by food}

Operant responding maintained by food was evaluated in mouse operant chambers (Model ENV-307A-CT, Med Associates, Georgia, VT, USA). The chambers were made of aluminum and acrylic, had grid floors (EVV-414, Med. Associates Inc., St Albans, USA), and were housed in sound and light-attenuated boxes equipped with fans to provide ventilation and white noise. The chambers were equipped with two retractable 
levers, one randomly selected as the active and the other as the inactive. Pressing on the active lever resulted in a pellet delivery (standard, fat-enriched pellet or high palatable) together with a stimulus-light during two sec (associated-cue), while pressing on the inactive lever had no consequences. A food dispenser equidistant between the two levers permitted delivery of food pellets when required. The beginning of the each operant responding session was signaled by turning on a house light placed on the ceiling of the box for three sec that was then turn off during the remaining duration of the session. The side of the active and inactive lever was counterbalanced between animals. Each session started with a priming delivery of one pellet. A time-out period of $10 \mathrm{sec}$ was established after each pellet delivery. During this period, the cue-light was off and no reinforcer was provided after responding on the active lever. Responses on the active lever and all the responses performed during the time-out period were also recorded. The session was terminated after 100 reinforcers were delivered or after one hour, whichever occurred first. One hour daily sessions were conducted seven days per week during a period of 41 days. The animals were food deprived five days before starting sessions to maintain their weight at $90 \%$ of their ad libitum initial weight adjusted for growth, and this food restriction regime was maintained during the first 10 sessions of the operant behavior training to permit the appropriate acquisition of the task. Additional standard- and high palatable-yoked groups were included. These groups were subjected to the same experimental conditions except that no reinforcer and no cue-light were presented after pressing in any of the two levers exposed in the operant chamber. However, standard- and high palatable-yoked groups received passively the same amount of pellets and with the same frequency as the $\mathrm{CB} 1+/+$ that were trained to obtain them. 
The training sessions started with a fixed-ratio 1 (FR1) schedule of reinforcement during 15 days (during the first 10 days mice were food deprived) where one active lever-press resulted in one food pellet. A minimum of $12 \mathrm{CB} 1+/+$ on each experimental group (included yoked-high palatable animals) were sacrificed after the exposure to the $10^{\text {th }}$ session, to evaluate the effects of short term exposure to different types of food on structural plasticity and molecular changes.

The rest of the animals were then feed ad libitum during the remaining operant behavior training. Operant training on FR1 was followed by a period of 10 days with a fixed ratio 5 (FR5) schedule (five active lever-presses were required to obtain one pellet). Mice were then exposed to a progressive ratio (PR) schedule in which the response requirement to earn one pellet escalated according to the following series: 1-2-3-5-1218-27-40-60-90-135-200-300-450-675-1000. The PR session lasted for four hours or until mice stopped responding for at least one hour, and was performed only once. The breaking point was determined in each animal as the last response ratio completed. Finally, mice were exposed to a FR5 schedule for 15 additional days. After each session, mice were returned to their home-cages. The chambers were cleaned at the end of each session to prevent the presence of odor of the previous mouse. Therefore, the schedule of the experiment is summarized as follows:

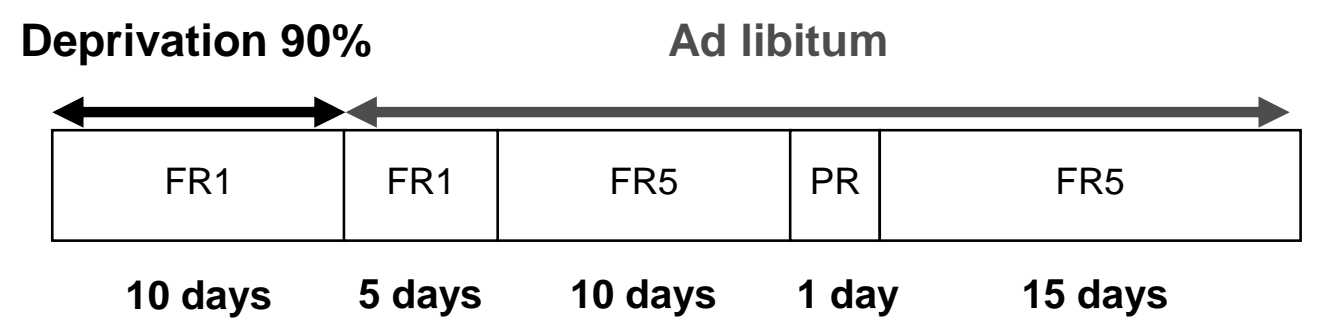


As previously described (Barbano et al., 2009), the criteria for the achievement of the operant responding were acquired when all of the following conditions were met: (i) mice maintained a stable responding with less than $20 \%$ deviation from the mean of the total number of reinforcers earned in three consecutive sessions (80\% of stability); (ii) at least $75 \%$ responding on the active hole, and (iii) a minimum of 10 reinforcers per session. All the mice included in this study have achieved these criteria in all the experimental phases.

\subsection{Food pellets}

During the operant experimental sessions, animals were presented with $20 \mathrm{mg}$ dustless precision standard pellets (TestDiet, Richmond, IN, USA), high palatable pellets (TestDiet, Richmond, IN, USA) or fat-enriched pellets (Bio-serv, Frenchtown, NJ, USA). The standard pellet formula was similar to the standard maintenance diet provided to mice in their home cage $(24.1 \%$ protein, $10.4 \%$ fat, $65.5 \%$ carbohydrate, with a caloric value of $3.30 \mathrm{kcal} / \mathrm{g}$ ). High palatable pellets presented similar caloric value to standard pellets $(20.5 \%$ protein, $12.7 \%$ fat, $66.8 \%$ carbohydrate, with a caloric value of $3.48 \mathrm{kcal} / \mathrm{g}$ ) with some differences in their composition. Thus, high palatable pellets were modified by the addition of chocolate flavor (2\% pure unsweetened cocoa), and although the carbohydrate content was similar in standard $(65.5 \%)$ and high palatable pellets $(66.8 \%)$, the proportion of sugars within this carbohydrate content was different: sucrose content in high palatable pellets was 50.11 $\%$ of the total carbohydrates, whereas in standard pellets was only $3.09 \%$. This different composition together with the cocoa content made high palatable pellets much more palatable than standard pellets. Finally, we also used a high caloric fat-enriched formula (14\% protein, $60 \%$ fat, $26 \%$ carbohydrate, with a caloric value of $5.32 \mathrm{kcal} / \mathrm{g}$ ). 
These pellets were presented only during the operant behavior sessions. Otherwise, animals were maintained on standard chow for their daily food intake.

\subsection{Ballistic Labeling with the Fluorescent Dye DiI}

See supplementary experimental procedures.

\subsection{Dendritic Spine Analysis}

Individual medium spiny neurons in the NAc and pyramidal neurons from the mPFC were chosen for spine analysis based on several criteria, as described previously (Lee et al., 2006): (i) there was minimal or no overlap with other labeled cells to ensure that processes from different cells would not be confused, (ii) at least three primary dendrites needed to be visible for cells to be used for analysis and (iii) distal dendrites (from secondary dendrites to terminal dendrites) were examined. Dendrites from medium spiny neurons in the core and shell of the NAc (from bregma 1.54 to bregma 1.10) and basilar dendrites of pyramidal neurons taken predominantly from the prelimbic and infralimbic areas of the $\mathrm{mPFC}$ (from bregma 1.98 to bregma 1.70) were analyzed.

To calculate spine density, a length of dendrite (at least $20 \mu \mathrm{m}$ long) was traced by using a confocal microscope (Zeiss LSM 510, Germany) with an oil immersion lens (40x). All images of dendrites were taken at different $\mathrm{z}$ levels $(0.3 \mu \mathrm{m}$ depth intervals $)$ to examine the morphology of dendritic spines. All measurements were made using IMAGE $\mathbf{J}$ analysis software. Protrusions from dendrites were classified into five types based on their morphology: class 1 protrusions, also called stubby protuberances were $0.5 \mu \mathrm{m}$ in length, lacked a large spine head, and did not appear to have a neck; class 2 , or mushroom-shaped spines were between 0.5 and $1.25 \mu \mathrm{m}$ long and were characterized 
by a short neck and large spine head; class 3, or thin spines ranged between 1.25 and 3.0 $\mu \mathrm{m}$ and had elongated spine necks with small heads; class 4, or wide spine were between 0.5 and $1.25 \mu \mathrm{m}$ long and were characterized by a large neck and a large spine head; and class 5 or branched spine ranged between 1.25 and $3.0 \mu \mathrm{m}$ and had elongated spine necks with two or more spine heads. Quantification of dendritic spine densities was performed in blind conditions.

\subsection{Acute bilateral intraNAc microinjection of rimonabant}

See supplementary experimental procedures.

2.7. Design and Construction of AAV Vectors and Viral production and purification

See supplementary experimental procedures.

\subsection{Bilateral intraNAc administration of AAV-scrambled or AAV-shCB1}

See supplementary experimental procedures.

\subsection{Tissue preparation and immunofluorescence analysis}

See supplementary experimental procedures.

\subsection{Immunoblot analysis}

See supplementary experimental procedures. 


\subsection{Statistical analysis}

The number of food pellets consumed and the active responses during the time-out period were analyzed by repeated measures two-way ANOVA with genotype (between subjects) and day (within subjects) as factors of variation, followed by corresponding post-hoc analysis when required (Dunnett's test).

Immunoblot data were analyzed by one-way ANOVA between subjects. Structural plasticity data were analyzed by a two-way ANOVA with genotype and kind of food as between subject factors of variation, followed by corresponding post-hoc analysis when required (Dunnett's test). An additional one-way ANOVA between subjects was used to compare yoked $\mathrm{CB} 1+/+$ receiving high palatable pellets non-contingently with other groups of $\mathrm{CB} 1+/+$ followed by the corresponding post-hoc Dunnett's analysis when required. All data were analyzed with SPSS software and are expressed as mean \pm SEM. Differences were considered significant at $\mathrm{p}<0.05$. 


\section{Results}

\subsection{Operant behavior to obtain high palatable food modifies seeking behavior}

through a CB1-R dependent mechanism.

Caloric content and palatability are two important properties of food involved in compulsive food taking (Johnson and Kenny, 2010; Davis and Carter, 2009). The endogenous cannabinoid system, through the activation of CB1-R, plays a key role in modulating food hedonic value. Therefore, we evaluated the behavioral consequences of prolonged training to obtain palatable food and the possible involvement of CB1-R in these responses. $\mathrm{CB} 1-/-$ and $\mathrm{CB} 1+/+$ were trained in an operant paradigm to obtain different kinds of food pellets: control standard diet $(3.30 \mathrm{kcal} / \mathrm{g})$, high caloric food rich in fat (high-fat pellets, $5.32 \mathrm{kcal} / \mathrm{g}$ ) or an isocaloric high palatable food (high palatable pellets, $3.48 \mathrm{kcal} / \mathrm{g}$ ). In fasted conditions, CB1-/- showed a decrease in the consumption and operant responding for standard, high-fat and high palatable pellets compared with CB1+/+ (Fig. 1A, B and C). The role of the endocannabinoid system regulating food intake and energy balance in central and peripheral tissues (Cota et al., 2003) could explain these differences (see discussion section).

In fed conditions, no differences in the operant behavior to obtain standard pellets were observed between genotypes (Fig. 1A). However, a decrease in the number of leverpresses to obtain high palatable pellets was observed during the training sessions in fed conditions in $\mathrm{CB} 1-/-$ when compared to $\mathrm{CB} 1+/+$ (Fig. 1C). A progressive ratio (PR) schedule was used to evaluate the motivation of mice for each type of food. Motivation of $\mathrm{CB} 1+/+$ to obtain this palatable food was significantly higher than for standard or high-fat pellets (Fig. 1D). CB1-/- trained to obtain high-fat pellets showed an enhancement in the number of lever-presses compared with $\mathrm{CB} 1+/+$ (Fig. 1B). In agreement, CB1-/- also showed an increased response for this type of food in the 
progressive ratio paradigm when compared to $\mathrm{CB} 1+/+$ (Fig. 1D). This last difference might be due to the caloric deficit produced by the deletion of the CB1-R since these animals present a lower amount of fat content (Cota et al., 2003) (see discussion section).

The number of lever-presses during the time-out periods that had no rewarding consequences was also evaluated as an indirect measure of food-seeking behavior that can be related to enhanced impulsivity. Training for high palatable pellets, but not for other types of food, increased the number of lever-presses during the time-out period at the beginning of the training period on fixed ratio (FR) 5 in $\mathrm{CB} 1+/+$. This response was further enhanced the last day of training on FR5 when compared with the initial training (Fig. 2), suggesting that palatable food progressively enhances this impulsive-like behavior developed during the training period. In contrast, this behavioral consequence of palatable food training was not observed in CB1-/-, which showed similar responses during the time-out periods when trained for the different kinds of food (Fig. 2).

We also evaluated the time course changes in body weight in both genotypes during the entire experiment. The lean phenotype previously reported in CB1-/- (Cota et al., 2003) was mainly maintained in this study in CB1-/- trained to obtain standard food, although weight differences between genotypes were attenuated in the groups trained to obtain high fat or high palatable pellets (data not shown). These results rule out a possible influence of differences in body weight in the changes in lever-pressing and food consumption observed between genotypes.

These results demonstrate that the high palatability of food, but not its caloric content, is associated with an enhanced motivation of mice to obtain food that progressively leads to increased seeking behavior, which can be related to enhanced impulsivity. These 
responses were absent in CB1-/- showing that the endocannabinoid system plays a critical role in this behavioral alteration.

\subsection{Operant behavior to obtain high palatable food modifies structural plasticity in the brain reward circuit.}

Long-lasting behavioral changes induced by drugs of abuse have been related to persistent changes in dendritic spines density in key areas of the brain reward system, such as the NAc core, NAc shell and the mPFC (Russo et al., 2010). We therefore evaluated whether operant behavioral training to obtain standard, high-fat or high palatable pellets could also induce structural neuroplastic changes in these brain structures. Immediately after the last training session, animals were sacrificed and their brains processed for ballistic delivery to label whole neurons with the dye DiI. We did not observe any modification in dendritic spine density in the NAc shell, core or mPFC of $\mathrm{CB} 1+/+$ and $\mathrm{CB} 1-/-$ trained on the operant lever-pressing to obtain standard food (Fig. 3A and B). In contrast, operant training to obtain high palatable pellets increased dendritic spine density in $\mathrm{CB} 1+/+$ in the NAc shell and in a lesser extent in the mPFC (Fig. 3A and B). Interestingly, these structural changes were not observed in the $\mathrm{CB} 1+/+$ yoked-high palatable group (Fig. 3D) that consumed similar amount of palatable pellets than their $\mathrm{CB} 1+/+$ master-paired mice (Fig. 3C), revealing that this response was selectively due to the operant training to obtain palatable food, and not to the passive exposure to this kind of pellets. Moreover, no differences in spine density in any of the brain areas investigated were observed between $\mathrm{CB} 1+/+$ trained to leverpress for standard pellets and their yoked-standard counterparts (Fig. 3D) in spite of the different operant performance of these two groups (Fig. 3C). These data demonstrate that the operant training by self and the associated motor responses were not correlated 
to the modifications in spine density observed in $\mathrm{CB} 1+/+$. In addition, these plasticity changes were absent in mice exposed to a short training period (10 days), demonstrating that are selectively produced by repeated food operant training (Fig. 3E).

Interestingly, a significant correlation was observed in $\mathrm{CB} 1+/+$ in the last training session between the changes in dendritic spine densities in the NAc shell and mPFC and the number of pellets consumed as well as the number of lever-presses during the timeout period (Fig. 4). Unlike the NAc shell and the mPFC, no modifications in dendritic spine density were observed in the NAc core (Fig. 3B), demonstrating differential adaptive changes produced by operant behavior to obtain palatable food in both subregions of the NAc. Therefore, training to obtain palatable food produced morphological changes in restricted areas of the brain reward circuit that are correlated to the enhanced seeking behavior produced by this operant training in $\mathrm{CB} 1+/+$.

Dendritic spines are classified into different groups depending on their morphology (stubby, thin, branched, wide and mushroom-type spines) and can undergo remodeling, that modifies their functionality (Bourne and Harris, 2007). Therefore, we also investigated the types of dendritic spines that were modified by operant behavior to obtain palatable food. An increase in the density of thin spines in the NAc shell, core and mPFC was observed in $\mathrm{CB} 1+/+$ trained to obtain palatable food, but not other kinds of food (Fig. 5). These changes were absent in CB1-/- trained to obtain high palatable food. CB1-/- only showed an enhanced density of branched spines in the NAc when trained with high-fat or high palatable pellets (Fig. 5). In addition, we also observed a small increase in the density of branched spines in the NAc shell and mPFC as well as in mushroom spines in the NAc shell in $\mathrm{CB} 1+/+$ trained with high palatable pellets (Fig. 5 and Table 1). These results demonstrate that operant training to obtain high palatable food produces structural neuroplastic changes due to the modification of specific types 
of dendritic spines, mainly thin spines, in the brain reward circuit. These structural changes selectively occur in mice showing enhanced seeking behavior after operant training.

\subsection{CB1-R in the nucleus accumbens mediates the behavioral and structural changes promoted by high palatable food.}

Our previous results suggest that CB1-R activity in the mesocorticolimbic system might regulate palatable food-induced behavioral and neuroplastic changes. We therefore evaluated the effects produced on these changes by the local inhibition of CB1-R in the NAc, the brain area where palatable food produced the strongest neuroplastic alterations. For this purpose, we first used an AAV9-shCB1 approach to knock-down the expression of $\mathrm{CB} 1-\mathrm{R}$ in the NAc of $\mathrm{CB} 1+/+$. The bilateral injection of AAV9shCB1 produced an inhibition of $50 \%$ of the total $\mathrm{CB} 1-\mathrm{R}$ in this brain area in $\mathrm{CB} 1+/+$ (Fig. 6B). Under these experimental conditions, the inhibition produced by AAV9shCB1 was specific of neurons and did not occur in glial cells (Fig. 6C and D). Control mice receiving AAV9-scrambled into the NAc showed the same consumption of high palatable pellets during FR1 and FR5 training, as well as similar responding in the PR paradigm and the time-out periods (Fig. $6 \mathrm{E}, \mathrm{F}$ and $\mathrm{G}$ ) than $\mathrm{CB} 1+/+$ in the previous experiment (Fig. 1C and D and Fig. 2). AAV9-shCB1 treated mice showed a decrease in the consumption of high palatable food (Fig. 6E), the breaking point response in the PR paradigm (Fig. 6F) and the changes promoted by palatable food on the lever-presses during the time-out periods (Fig. 6G) in comparison with AAV9-scrambled control animals.

We also evaluated the effects of an acute administration of the selective CB1-R antagonist rimonabant locally into the NAc of $\mathrm{CB} 1+/+$. In agreement with the previous 
results, rimonabant bilaterally microinjected into the NAc at the lowest dose $(1 \mu \mathrm{g} / \mathrm{site})$ selectively decreased high palatable pellet consumption (Fig. 7A). Higher doses of rimonabant produced non-specific effects that were not revealed when using the genetic approach in the previous experiments (see Fig. 1), and also decreased the consumption of other kinds of pellets (Fig. 7A). Taken together the results of both experimental approaches, we clearly demonstrate the selective involvement of CB1-R in the NAc in the behavioral responses induced by palatable food. These behavioral results obtained after down-regulation of CB1-R in the NAc were similar to those obtained in CB1-/-, ruling out possible compensatory mechanism in the knockout mice that could interfere with these responses.

Down-regulation of CB1-R in the NAc by AAV9-shCB1 also attenuated the changes promoted by palatable food in dendritic spine density in the NAc shell that were revealed in control AAV9-scrambled mice. Indeed, the dendritic spine morphology in these control mice (Fig. $6 \mathrm{H}$, I and J) was similar than in $\mathrm{CB} 1+/+$ in the previous experiment (Fig. 3). This attenuation was mainly due to a decrease in the density of thin, branched and mushroom subtypes of spines (Fig. 6J). These results and the similarities to those previously revealed in CB1-/- demonstrate the specific involvement of CB1-R in the NAc in food-induced neuroplastic changes, and the cause-relationship between behavioral and morphological changes induced by palatable food. 


\section{Discussion}

In this study, we revealed for the first time that the hedonic value of food, but not its caloric content, modifies structural plasticity in restricted areas of the corticolimbic system. These morphological changes selectively occur in mice showing enhanced food-seeking responses and associated impulsivity-like behavior. Using knock-out, pharmacological and viral approaches, we also demonstrated that CB1-R are necessary to produce these behavioral and morphological changes induced by reinforced learning with palatable food.

In agreement with previous studies (see for review Saper et al., 2002), we observed that food palatability strongly promotes operant behavior and represents the key factor for food motivation. Moreover, mice working for palatable pellets progressively increased their operant responses even when no reward can be obtained, suggesting an enhancement of food-seeking behavior that can be related to an enhanced impulsivity. Accordingly, escalation of palatable food seeking behavior has been correlated with elevated impulsivity (Diergaarde et al., 2009), and intermittent access to this kind of food leads to behavioral and neurochemical changes that resemble those produced by drugs of abuse (Avena et al., 2008; Belin et al., 2008). Therefore, chronic exposure to palatable food enhances reward-related seeking responses and impulsive-like behavior, which could account for progressive food overconsumption and the development of food-seeking and binge-eating. Our operant paradigm is a useful model to reveal these behavioral alterations induced by palatable food and to study their neurobiological substrate. A behavioral alteration leading to loss of control and addictive-like behavior has also been recently reported in rodents exposed to high caloric food (Johnson and Kenny, 2010). 
Repeated exposure to drugs of abuse modifies structural plasticity in the brain reward system, which may underlie some addictive behaviors (Russo et al., 2010). Previous studies have mainly focused on the morphology of dendritic spines since the majority of excitatory synaptic inputs involved in neuroplastic modifications occur in these structures (Harris and Kater, 1994). We therefore evaluated whether plastic changes similar to those produced by drugs of abuse also occurred in mice exposed to the different kinds of food in our operant paradigm. We found that operant training with palatable food increased spine density in the NAc shell and in a lesser extent in the mPFC, two brain regions crucial for regulating the hedonic aspects of food (Baldo and Kelly, 2007; Mahler et al., 2007), decision-making and impulsive responses (Volkow and Fowler, 2000). Interestingly, similar structural changes induced by chronic exposure to psychostimulants or nicotine have been hypothesized to participate in the development of addictive behavior, although conflicting results have been recently reported (Russo et al., 2010).

We hypothesize that the increased spine density observed in the NAc shell and mPFC might be responsible for the abnormal behavioral responses observed in mice trained to obtain palatable pellets, including enhanced seeking-behavior and related impulsivity. Overtraining and the motor responses associated to operant training were not responsible of the changes in dendritic spine densities observed in $\mathrm{CB} 1+/+$ since no differences in structural plasticity were observed between $\mathrm{CB} 1+/+$ trained for standard pellets and their corresponding yoked animals, in spite of the different operant performance of these two groups. Previous studies did not observe changes in structural plasticity 30 days after operant training with palatable food in rats (Crombag et al., 2005). A possible explanation for this discrepancy could be the different animal species or the transitory nature of the plasticity change promoted by palatable food, although 
further studies are required to clarify the duration of this change. This would be in contrast with the long-lasting alterations in neuronal morphology observed after the exposure to drugs of abuse (Russo et al., 2010).

A contingency between the operant behavior and the delivery of the reward was necessary for the induction of the neuroplastic changes promoted by palatable food since these modifications were not observed in mice that received palatable pellets passively and consuming the same amount of pellets (Fig. 3). This is also in contrast with drugs of abuse since psychostimulants enhanced dendritic spine density in the mesocorticolimbic system in rodents that received contingently the drug, but also to a lesser extent in animals that received passively the drug (Russo et al., 2010).

In contrast to the NAc shell, only minor morphological changes were observed in the NAc core, suggesting that these two structures are involved in different aspects of feeding behavior. In this sense, the NAc core plays a more prominent role in foodinduced goal-directed exploratory behaviors (Cardinal et al., 2002), whereas the NAc shell is more directly related to the hedonic value of food (Peciña and Berridge, 2005). However, drugs of abuse also extensively modify dendritic spine density in the NAc core (Russo et al., 2010), suggesting that morphological alterations in this region might play a more prominent role in mediating the effects of drugs of abuse than palatable food (McFarland et al., 2003).

We have also investigated the possible involvement of $\mathrm{CB} 1-\mathrm{R}$ in the behavioral and morphological effects of palatable food considering the crucial role of these receptors in food hedonic value (Mahler et al., 2007). The constitutive deletion of CB1-R decreased operant behavior and the motivation to obtain palatable food, without affecting the operant responses to obtain standard pellets. The behavioral alterations produced by palatable food in $\mathrm{CB} 1+/+$ were also absent in $\mathrm{CB} 1-/-$. To evaluate the specific 
participation of CB1-R in the NAc and to rule out possible compensatory changes in the CB1-/-, we used pharmacological and viral approaches to locally decrease CB1-R activity in the NAc in $\mathrm{CB} 1+/+$. The acute pharmacological blockade of CB1-R in the NAc as well as the chronic down-regulation of these receptors by intraNAc microinjection of AAV9-shCB1 decreased the behavioral (pharmacological and viral approaches) and structural plasticity (viral approach) responses induced by palatable food in a similar manner that it was observed in CB1-/-. These results demonstrate the specific involvement of CB1-R in the NAc in these behavioral and morphological changes promoted by palatable food. Interestingly, other studies have reported that the endogenous cannabinoid system regulates behavioral aspects related to the loss of control over drug intake (Maldonado et al., 2006), and modulates impulsivity in a stop signal task (McDonald et al., 2003). Our results extend these observations and suggest that $\mathrm{CB} 1-\mathrm{R}$ also modulate seeking behavior and related impulsivity promoted by the exposure to palatable food. On the other hand, CB1-/- showed an enhanced operant response and motivation to obtain high-fat pellets. This observation agrees with several studies showing that CB1-/- have a lower amount of fat content (Cota et al., 2003) and would require more caloric intake than $\mathrm{CB} 1+/+$. A decreased lipid storage in adipocytes in CB1-/- might be responsible (Cota et al., 2003), at least in part, of the lean phenotype and increased high fat pellet consumption observed in these knockout animals. In addition, a recent study has shown that endocannabinoid-induced CB1-R activation in the small intestine contributes to orosensory-mediated induction of fat intake (DiPatrizio et al., 2011). Our results and those recent findings support the role of CB1-R in the regulation of high caloric food intake.

The acquisition of operant responding is a learning/memory process that can lead to the change of the shape and structure of the dendritic spines, which also modifies their 
functionality (Bourne and Harris, 2007). In agreement, predominant enhancement in the proportion of thin spines in the NAc shell and mPFC was observed in mice trained to obtain palatable food, but not other kinds of food. These changes were mediated by CB1-R in the NAc since they were absent in CB1-/- and CB1+/+ receiving AAV9shCB1 into this brain structure. Thin spines are considered transient and highly plastic structures that can both stabilize and transform into more mature spines in response to behavioral changes and during learning processes by incorporating AMPA receptors (Bourne and Harris, 2007) or can shrink and finally disappear. Hence, the enhancement of thin spines induced by operant training to obtain palatable food could increase the strength of specific synapses in the NAc shell and mPFC, and promote the behavioral abnormalities observed during this operant training. Although operant training with palatable food mainly enhanced the density of thin spines, the microinjection of AAV9shCB1 into the NAc also decreased the density of other kind of spines. Thus, the generation of stabilized spines appears to represent a plasticity change associated to palatable food operant learning with the participation of CB1-R.

In conclusion, we reveal behavioral alterations promoted by operant training to obtain palatable food that are associated to neuroplastic changes. Similar morphological alterations have been reported in these brain structures after exposure to drugs of abuse, which play a crucial role in addictive behavior. Therefore, these neural changes induced by palatable food could represent a biological substrate to explain some of the alterations in food-seeking behavior that could eventually lead to eating-related disorders and promote obesity. Our results also provide an important advance in the understanding of the common links between food-seeking and drug addiction. 


\section{References}

Avena, N., Rada, P., Hoebel, B.G., 2008. Evidence for sugar addiction: Behavioral and neurochemical effects of intermittent, excessive sugar intake. Neurosci \& Biobehav Rev. 32, 20-39.

Ayuso, E., Mingozzi, F., Montane, J., Leon, X., Anguela, X.M., Haurigot, V., Edmonson, S.A., Africa, L., Zhou, S., High, K.A., Bosch, F., Wright, J.F., 2010. High AAV vector purity results in serotype- and tissue-independent enhancement of transduction efficiency. Gene Ther. 17, 503-510.

Baldo, B.A., Kelley, A.E., 2007. Discrete neurochemical coding of distinguishable motivational processes: insight from nucleus accumbens control of feeding. Psychopharmacology (Berl). 191, 439-459.

Ballesteros-Yáñez, I., Valverde, O., Ledent, C., Maldonado, R., DeFelipe, J., 2007. Chronic cocaine treatment alters dendritic arborization in the adult motor cortex through a CB1 cannabinoid receptor-dependent mechanism. Neuroscience. 8, 15361545.

Barbano, M.F., Castañe, A., Martin-Garcia, E., Maldonado, R., 2009. Delta-9tetrahydrocannabinol enhances food reinforcement in a mouse operant conflict test. Psychopharmacology. 205, 475-487.

Belin, D., Mar, A.C., Dalley, J.W., Robbins, T.W., Everitt, B.J., 2008. High impulsivity predicts the switch to compulsive cocaine-taking. Science. 320, 1352-1355.

Bertran-Gonzalez, J., Bosch, C., Maroteaux, M., Matamales, M., Herve, D., Valjent, E., Girault, J.A., 2008. Opposing patterns of signallling activation in dopamine D1 and D2 receptor-expressing striatal neurons in response to cocaine and haloperidol. J Neurosci. 28, 5671-5685. 
Bourne, J, Harris, K.M., 2007. Do thin spines learn to be mushroom spines that remember?. Curr Opin Neurobiol. 17, 381-386.

Caballero, R., 2007. The global epidemic of obesity: an overview. Epidemiol Rev. 29, $1-5$.

Cardinal, R.N., Parkinson, J.A., Lachenal, G., Halkerston, K.M., Rudarakanchana, N., Hall, J., Morrison, C.H., Howes, S.R., Robbins, T.W., Everitt, B.J., 2002. Effects of selective excitotoxic lesions of the nucleus accumbens core, anterior cingulated cortex, and central nucleus of the amygdala on autoshaping performance in rats. Behav Neurosci. 116, 553-56.

Cearley, N., Vandenberghe, L.H., Parente, M.K., Carnish, E.R., Wilson, J.M., Wolfe, J.H., 2008. Expanded Repertoire of AAV Vector Serotypes Mediate Unique Patterns of Transduction in Mouse Brain. Molecular Therapy. 16, 1710-1718.

Cota, D., Marsicano, G., Tschöp, M., Grübler, Y., Flachskamm, C., Schubert, M., Auer, D., Yassouridis, A., Thöne-Reineke, C., Ortmann, S., Tomassoni, F., Cervino, C., Nisoli, E., Linthorst, A.C., Pasquali, R., Lutz, B., Stalla, G.K., Pagotto, U., 2003. The endogenous cannabinoid system affects energy balance via central orexigenic drive and peripheral lipogenesis. J. Clin. Invest. 112, 423-431.

Crombag, H.S., Gorny, G., Li, Y., Kolb, B., Robinson, T.E., 2005. Opposite effects of amphetamine self-administration experience on dendritic spines in the medial and orbital prefrontal cortex. Cereb Cortex. 15, 341-348.

Davis, C., Carter, J.C., 2009. Compulsive overeating as an addiction disorder. A review of theory and evidence. Appetite. 53, 1-8.

Di Marzo, V., Matias, I., 2005. Endocannabinoid control of food intake and energy balance. Nat Neurosci. 8, 585-589. 
DiPatrizio, N.V., Astarita, G., Schwartz, G., Li, X., Piomelli, D., 2011. Endocannabinoid signal in the gut controls dietary fat intake. Proc Natl Acad Sci USA. 108, 12904-12908.

Diergaarde, L., Pattij, T., Nawijn, L., Schoffelmeer, A.N., De Vries, T.J., 2009. Trait impulsivity predicts escalation of sucrose seeking and hypersensitivity to sucroseassociated stimuli. Behav Neurosci. 123, 794-803.

Grutzendel, J., Tsai, J., Gan, W.B., 2003. Rapid labelling of neuronal populations by ballistic delivery of fluorescent dyes. Methods. 30, 79-85.

Harris, K.M., Kater, S.B., 1994. Dendritic spines: cellular specializations imparting both stability and flexibility to synaptic function. Annu Rev Neurosci. 17, 341-371.

Johnson, P.M., Kenny, P.J., 2010. Dopamine D2 receptors in addiction-like reward dysfunction and compulsive eating in obese rats. Nat. Neurosci. 13, 635-641.

Khan, L.K., Sobush, K., Keener, D., Goodman, K., Lowry, A., Kakietek, J., Zaro, S., Moore, J., Dunet, D., Galuska, D. et al., 2009. Recommended community strategies and measurements to prevent obesity in the United States. MMWR Recomm Rep. $58,1-26$.

Lee, K.W., Kim, Y., Kim, A.M., Helmin, K., Nairn, A.C., Greengard, P., 2006. Cocaine-induced dendritic spine formation in D1 and D2 dopamine receptorcontaining medium spiny neurons in nucleus accumbens. Proc Natl Acad Sci USA. 103, 3399-3404.

Li, Y., Acerbo, M.J., Robinson, T.E., 2004. The induction of behavioural sensitization is associated with cocaine-induced structural plasticity in the core (but not shell) of the nucleus accumbens. Eur. J. Neurosci. 20, 1647-1654. 
Mahler, S.V., Smith, K.S., Berridge, K.C., 2007. Endocannabinoid hedonic hotspot for sensory pleasure: anandamide in nucleus accumbens shell enhances 'liking' of a sweet reward. Neuropsychopharmacology. 32, 2267-2278.

Maldonado, R., Valverde, O., Berrendero, F., 2006. Involvement of the endocannabinoid system in drug addiction. Trends Neurosci. 29, 225-232.

Mas, A., Montané, J., Anguela, X.M., Muñoz, S., Douar, A.M., Riu, E., Otaegui, P., Bosch, F., 2006. Reversal of type 1 diabetes by engineering a glucose sensor in skeletal muscle. Diabetes. 5, 1546-1553.

McDonald, J., Schleifer, L., Richards, J.B., de Wit, H., 2003,). Effects of THC on behavioral measures of impulsivity in humans. Neurophychopharmacology. 28, 1356-1365.

McFarland, K., Lapish, C.C., Kalivas, P.W., 2003. Prefrontal glutamate release into the core of the nucleus accumbens mediates cocaine-induced reinstatement of drugseeking behavior. J Neurosci. 15, 3531-3537.

Moeller, F.G., Dougherty, D.M., Barratt, E.S., Schmitz, J.M., Swann, A.C., Grabowski, J., 2001. The impact of impulsivity on cocaine use and retention in treatment. J Subst Abuse Treat. 21, 193-198.

Nederkoorn, C., Braet, C., Van Eijs, Y., Tanghe, A., Jansen, A., 2006. Why obese children cannot resist food: the role of impulsivity. Eat Behav. 7, 315-322.

Peciña, S., Berridge, K.C., 2005. Hedonic hot spot in nucleus accumbens shell: where do mu-opioids cause increased hedonic impact of sweetness?. J Neurosci. 25, 11777-11786.

Paxinos, G., Franklin, K.B.J., 2001. The mouse brain in stereotaxic coordinates. Academic press, San Diego, USA. 
Pitchers, K.K., Balfour, M.E., Lehman, M.N., Richtand, N.M., Yu, L., Coolen, L.M., 2010. Neuroplasticity in the Mesolimbic System Induced by Natural Reward and Subsequent Reward Abstinence. Biol Psychiatry. 67, 872-879.

Puighermanal, E., Marsicano, G., Busquets-Garcia, A., Lutz, B., Maldonado, R., Ozaita, A., 2009. Cannabinoid modulation of hippocampal long-term memory is mediated by mTOR signaling. Nat. Neurosci, 12, 1152-1158.

Rosval, L., Steiger, H., Bruce, K., Israël, M., Richardson, J., Aubut, M., 2006. Impulsivity in women with eating disorders: problem of response inhibition, planning, or attention?. Int J Eat Disord. 39, 590-593.

Robinson, T.E., Gorny, G., Mitton, E., Kolb, B., 2001. Cocaine self-administration alters the morphology of dendrites and dendritic spines in the nucleus accumbens and neocortex. Synapse. 39, 257-266.

Russo, S.J., Dietz, D.M., Dumitriu, D., Morrison, J.H., Malenka, R.C., Nestler, E.J., 2010. The addicted synapse: mechanisms of synaptic and structural plasticity in nucleus accumbens. Trends Neurosci. 756, 1-10.

Saper, C.B., Chou, T.C., Elmquist, J.K., 2002. The need to feed: homeostatic and hedonic control of eating. Neuron. 36, 199-211.

Tafuro, S., Ayuso, E., Zacchigna, S., Zentilin, L., Moimas, S., Dore, F., Giacca, M., 2009. Inducible adeno-associated virus vectors promote functional angiogenesis in adult organisms via regulated vascular endothelial growth factor expression. Cardiovasc Res. 83, 663-671.

Volkow, N.D., Fowler, J.S., 2000. Addiction, a disease of compulsion and drive: involvement of the orbitofrontal cortex. Cereb Cortex. 10, 318-325. 
Zimmer, A., Zimmer, A.M., Hohmann, A.G., Herkenham, M., Bonner, T.I., 1999. Increased mortality, hypoactivity and hypoalgesia in cannabinoid CB1 receptor knockout mice. Proc Natl Acad Sci USA. 96, 5780-5785. 


\section{FIGURE LEGENDS}

Figure 1 Highly palatable food strongly promotes operant behaviour and enhances motivation for food through a CB1-R dependent mechanism. (A) Daily consumption of standard pellets in $\mathrm{CB} 1+/+$ (black triangle; $\mathrm{n}=36$ ) and $\mathrm{CB} 1-/-($ white triangle; $\mathrm{n}=14$ ) mice. (B) Daily consumption of high caloric high-fat pellets in $\mathrm{CB} 1+/+$ (black rectangle; $n=29$ ) and CB1-/- (white rectangle; $n=13$ ). (C) Daily consumption of high palatable pellets in $\mathrm{CB} 1+/+$ (black circle; $n=37$ ) and $\mathrm{CB} 1-/$ - (white circle; $\mathrm{n}=17$ ). (D) Breaking point reached by $\mathrm{CB} 1+/+$ (black bars) and CB1-/- (white bars) for each kind of food in a progressive ratio paradigm, as a measure of motivation for food. Data are expressed as mean \pm s.e.m. $\star p p<0.05, \star \star p<<0.01, \star \star \star p<<0.001(\mathrm{CB} 1+/+\mathrm{vs}$

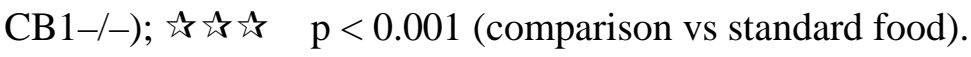

Figure 2 Operant training for palatable food increases impulsive-like behavior through a CB1-R dependent mechanism (A) Unrewarding responses on the active lever during the time-out period in the first and last day of the operant training in FR5 in CB1+/+ ( $\mathrm{n}=29-37$ mice per group) and CB1-/- (13-17 mice per group) trained for each kind of food, as a measure of food seeking behavior. (B) Time course diagram representing the number of unrewarding time-out active responses (every 5 days) throughout the entire experimental period on FR5 in $\mathrm{CB} 1+/+$ trained for standard (white bar), high fat (grey bar) and high palatable (black bar) pellets. (C) Time course diagram representing the number of unrewarding time-out active responses (every 5 days) throughout the entire experimental period on FR5 in CB1-l- trained for standard (white bar), high fat (grey bar) and high palatable (black bar) pellets. Data are expressed as mean \pm s.e.m. औえた p 
$<0.001$ (comparison vs standard food); \# p < $0.05 \#$ \# p < 0.01, \#\# \# p $<0.001$ (day 16 vs day 40).

Figure 3 Neuronal morphological changes induced by palatable food are mediated by a CB1R mechanism. (A) Illustration of DiI-labeled dendrites of medium spiny neurons in the NAc shell of $\mathrm{CB} 1+/+$ and $\mathrm{CB} 1-/-$ trained to obtain standard, high fat or high palatable pellets. (B) Total dendritic spines density in neurons from the NAc shell, core and mPFC of $\mathrm{CB} 1+/+$ (black bars) and CB1-/- (white bars) after repeated operant training to obtain standard, high-fat or high palatable pellets. Data represents the average of 7 to 8 mice per experiment group and a total of 6-12 dendrites per animal and brain area. No more than 2 dendrites were evaluated from the same neuron. (C) Number of pellets consumed and number of active lever pressings per hour in $\mathrm{CB} 1+/+$ trained to obtain standard or palatable pellets and their corresponding yoked-control groups ( $n=20-28$ animals per group). (D) Total dendritic spines density in neurons from the NAc shell, core and mPFC of $\mathrm{CB} 1+/+$ trained to obtain standard or palatable pellets and their corresponding yoked-control groups ( $n=7-9$ animals per group). A total of 612 dendrites per animal and brain area were evaluated. No more than 2 dendrites from the same neuron were included. (E) Total dendritic spine density in $\mathrm{CB} 1+/+$ exposed to operant training for standard, high caloric or high palatable food for a short period of 10 days ( $\mathrm{n}=6$ mice/group). A total of $6-12$ dendrites per animal and brain area were evaluated. No more than 2 dendrites from the same neuron were included. Data are expressed as mean \pm s.e.m. $\star \mathrm{p}<0.05(\mathrm{CB} 1+/+$ vs $\mathrm{CB} 1-/-)$; is $\mathrm{p}<0.05$, (comparison vs standard food); \# $\mathrm{p}<0.05$; \# \# $\mathrm{p}<0.01$, \# \# \# $\mathrm{p}<0.001$ (comparison between contingent and yoked control group). 
Figure 4 Changes in structural plasticity statistically correlate with changes in eating behaviour in $\mathrm{CB} 1+/+$. (A and B) Statistical correlation between the dendritic spines density in neurons of the NAc shell and the number of pellets consumed or the active operant responding during the time-out period in the last training session $(n=6-9$ animals per group). (C and D) Statistical correlation between the dendritic spines density in neurons of the mPFC and the number of pellets consumed or the active operant responding during the time-out period in the last training session $(n=6-9$ animals per group). $\mathrm{CB} 1+/+$ trained for standard pellets are represented by black triangles, $\mathrm{CB} 1+/+$ trained for high-fat pellets are represented by black squares and $\mathrm{CB} 1+/+$ trained for palatable pellets are represented by black circles.

Figure 5 Dendritic density of thin (A) and branched (B) spines in neurons of the NAc shell, core and mPFC in $\mathrm{CB} 1+/+$ (black bar) and $\mathrm{CB} 1-/-$ (white bar) trained to obtain standard, high fat or high palatable pellets and in $\mathrm{CB} 1+/+$ that received passively high palatable pellets (yoked high palatable). A total of 6 to 12 dendrites per animal and brain area and a total of 7-8 mice per experimental group were studied. A maximum of 2 dendrites per neuron were analyzed. Data expressed as mean \pm s.e.m. $\quad \star \quad p<0.05$, $\star \star \mathrm{p}<0.01(\mathrm{CB} 1+/+$ vs $\mathrm{CB} 1-/-)$ 方 $\mathrm{p}<0.05$, 动弥 $\mathrm{p}<0.01$ (comparison vs standard food); \# \# p < 0.01 (contingent high palatable vs yoked high palatable). 
Figure 6 Knock-down of $\mathrm{CB} 1-\mathrm{R}$ in the NAc in $\mathrm{CB} 1+/+$ prevents behavioral and neuronal morphological changes induced by palatable food. (A) Illustration of the NAc showing an example of the localization of the AAV9-injections. Green staining represents the localization and diffusion of AAV9 vector expressing GFP cDNA. (B) Immunoblots for CB1-R and GADPH of samples from the hippocampus (Hc) and NAc, and optical density quantification of CB1-R in samples from both brain areas. AAV9scrambled (SCR): $n=4-5$; AAV9-shCB1 (sh CB1): $\mathrm{n}=4-6$ mice. $(\mathbf{C}$ and D) Double immunofluorescence analysis showing the specific co-localization of GFP-positive cells (in green) with neurons (NeuN positive cells in red, co-localization in orange) but not with glial cells (Iba-1 (C) or GFAP (D) positve cells in blue); $n=4-6$ mice. (E) Daily consumption of high palatable pellets in mice receiving AAV9-scrambled $(n=20)$ and AAV9-shCB1 $(\mathrm{n}=20)$ into the NAc. (F) Breaking point for high palatable pellets in a progressive ratio paradigm reached by mice treated with AAV9-scrambled $(n=20)$ or AAV9-shCB1 ( $\mathrm{n}=20)$ into the NAc. (G) Unrewarding responses on the active lever during the time-out period in the first and last day of the operant training on FR5 in mice trained for high palatable pellets receiving AAV9-scrambled $(n=20)$ or AAV9shCB1 $(n=20)$ into the NAc. $(\mathbf{H})$ Illustration of DiI-labeled dendrites of medium spiny neurons in the NAc shell of CB1+/+ treated with AAV9-scrambled or AAV9-shCB1. (I) Total dendritic spines density in neurons from the NAc shell, core and mPFC of $\mathrm{CB} 1+/+$ treated with AAV9-scrambled (black bars) or AAV9-shCB1 (white bars). (J) Dendritic spine density of thin, branched, mushroom, stubby and wide spines in $\mathrm{CB} 1+/+$ treated with AAV9-scrambled (black bar) or AAV9-shCB1 (white bars). Data represents the average of 7 to 9 mice per experiment group and a total of 6-12 dendrites per animal and brain area. No more than 2 dendrites were evaluated from the same 
neuron. Data are expressed as mean \pm s.e.m. $\star \quad p<0.05, \star \star \quad p<0.01, \star \star \star \quad p<$ 0.001 (AAV9-scrambled vs AAV9-shCB1); \# \# p < 0.01 (day 16 vs day 40).

Figure 7 Acute inhibition of CB1-R activity in the NAc of CB1+/+ decreases operant responding and pellet consumption. (A) Histogram showing the total number of standard, high-fat and high palatable pellets consumed before treatment after surgery (mean of the last three sessions before treatment), and after bilateral injection into the NAc of vehicle or the different doses of rimonabant. $\mathrm{N}=6-9$ mice per experimental group. (B) Schematic diagrams adapted from the mouse brain atlas of Paxinos and Franklin (1997) showing representative injection sites of rimonabant in the NAc. Black dots indicate locations of cannula tips. Each image indicates the distance from Bregma.

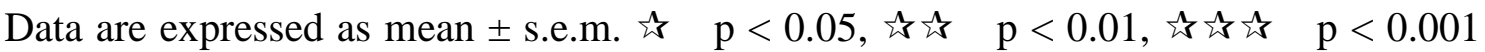
(comparison vs standard food); $\# \mathrm{p}<0.05 ; \# \mathrm{p}<0.01$ (vehicle vs different doses).

Table 1 Dendritic spine density of mushroom-, stubby- or wide-type of dendritic spines in $\mathrm{CB} 1+/+$ and $\mathrm{CB} 1-/-$. 